\title{
Strategies and Design Principles to Minimize Negative Side-effects of Digital Motivation on Teamwork
}

\author{
Abdullah Algashami, Alimohammad Shahri, John McAlaney, Jacqui Taylor, Keith \\ Phalp, Raian Ali \\ Faculty of Science and Technology \\ Bournemouth University, United Kingdom \\ \{aalgashami, ashahri, jmcalaney, jtaylor, kphalp, rali\}@bournemouth.ac.uk
}

\begin{abstract}
Digital Motivation in business refers to the use of technology in order to facilitate a change of attitude, perception and behaviour with regards to adopting policies, achieving goals and executing tasks. It is a broad term to indicate existing and emerging paradigms such as Gamification, Persuasive Technology, Serious Games and Entertainment Computing. Our previous research indicated risks when applying Digital Motivation. One of these main risks is the impact it can have on the interpersonal relationships between colleagues and their individual and collective performance. It may lead to a feeling of unfairness and trigger negative group processes (such as social loafing and unofficial clustering) and adverse work ethics. In this paper, we propose a set of strategies to minimize such risks and then consolidate these strategies through an empirical study involving managers, practitioners and users. The strategies are then analysed for their goal, stage and purpose of use to add further guidance. The strategies and their classification are meant to inform developers and management on how to design, set-up and introduce Digital Motivation to a business environment, maximize its efficiency and minimize its side-effects on teamwork.
\end{abstract}

Keywords: Digital Motivation, Persuasive Technology, Gamification, Motivation Engineering.

\section{Introduction}

Digital Motivation (hereafter DM) is on the rise and there exist already various established domains which characterize it including Persuasive Technology [1], Gamification [2], Games with Purpose [3] and Entertainment Computing [4]. Central to $\mathrm{DM}$ is the use of technology (including games and social computing), to prevent, change, maintain or enhance certain behaviours and attitudes in relation to certain policies, goals, tasks, and social inter-relations. The advances in technology, including mobile and sensing technology, and the increased familiarity of the public with advanced features of Web 2.0, games and social computing have made these techniques possible and acceptable. DM has been used in various application areas including health [5, 6], sport [7], sales [8] and education [9, 10]. 
There exist different methods and principles of developing DM. Fogg [11] proposes eight steps of developing and introducing Persuasive Technology. The emphasis in these steps is on the choice of behaviour, the audience and finally, understanding the obstacles. Nicholson [12] proposes a theoretical framework for a 'meaningful gamification' intended to avoid the risk of losing intrinsic motivation when gamifying tasks. Other principles are either focused on single property of DM or coupled with certain application areas. For example, Consolvo et al [13] focus on goal-setting and explore ways to elicit goals and specify their time frames. GramHansen [14] proposes an approach based on participatory design and constructive ethics to achieve a persuasive design.

We advocate that some DM techniques and methods have potential side effects on teamwork. In [15] we concluded that gamification solutions can cause social and mental well-being problems in the work place and that there is a need to consider ethics and values when adopting such solutions. Nicholson [16] argues that gamification can be seen as exploitation if implemented in certain ways that drive people to do more than their job description would imply. Timmer et al [17] focus their study on the importance of user-informed consent prior to the use of persuasion. This human aspect in relation to the potential side-effect suggests that we need to take it as an initial requirement when planning and engineering DM. However, while the focus of existing literature is on ways to develop successful DM, there exists little emphasis on how to engineer counter-measures to avoid these side-effects.

Issues that may arise as a result of introducing DM to the work space include reduced collegiality, negative group relations and low group cohesion. For example, introducing a leader-board to a collaborative workplace which is based on measuring individual performance could lead to less collaboration and introduce questions about the measurement of individuals' performances. Social recognition elements, e.g. badges and status, given to groups based on their collective performance may introduce a risk of social loafing [18] and a pressure for social compensation [19].

In our previous work [20] a reference model has been explored and developed, putting together the properties of motives, environment and users which are involved when taking decisions during the development and deployment of DM solutions. In [21], we developed various personas and argued that individual differences need to be catered for DM design and customization to maximize its acceptance and efficiency and also avoid the side-effects discussed in [15]. However, the design principles and tools for preventive and corrective mechanisms to deal with these potential issues of DM have not yet been explored.

In this paper, we build on our previous results presented in [15, 20, 21] and identify strategies that DM development and management can adopt to introduce DM into the workplace with the aim of minimizing the risks it may introduce into teamwork. As a method, we further analyse the results of our previous works and review the literature to come up with an initial set of strategies. This set is then discussed and elaborated in interviews with managers, practitioners and users. A focus group to confirm and categorize the results was then conducted. The results of this paper will be beneficial for developers, management and occupational psychologists to avoid negative experiences DM can facilitate if introduced without careful considerations. 


\section{Motivating Scenario}

We will present two cases to illustrate how an ad-hoc introduction of DM could affect the efficiency of the teamwork environment. In the first case, we highlight workplace intimidation. In the IT department of a company, the front-end development team is responsible for ensuring that the user experience (UX) is kept at a satisfactory level, and also responsible for updating the user interface (UI) when necessary to address customers' requirements. Collaboration of the team members is crucial to the success of the department's work and failure to maintain appropriate communication and collaboration might affect the quality of the final artefact. The UI has great value for the company as they believe this is the client view of the company. Therefore, the company wishes to decrease the chance of failure in the design of the UI as much as possible. Thus, in order to encourage collaboration, the organisation using status as a DM technique to motivate the front-end development team based on its overall performance. For communication and tracking purposes, team members have access to individuals' work performance. This could help them to schedule plans and make changes more easily if needed. However, since team members have access to each other's performance details, there is a risk of negative effect in the group. Team members with better performance may feel closer to each other causing groups to form, and this may pave the way for workplace intimidation, where some highperformance employees bully lower-performance colleagues in the team. This illustrates how using DM might create tension or conflict amongst workers and the need to have strategies to resolve such negative effect.

The second case involves a situation where sabotage could happen within teamwork in the workplace. Two teams are working in an IT department creating a web application. John, Alice and Bob are team A and are working on the design of the UI while Mary, James and Matt are team B and responsible for the back-end development. The manager asks team A to update the design of the UI in a specific time-frame. Bob calls in sick and does not attend work for two weeks. The manager delegates his work to Alice from team B. The department, which uses a leader-board, as a DM technique, to encourage both teams to finish their tasks on time, decides to give points to the team who can finish the task on-time. At the end, the team with most points will receive a reward. Since Alice is from team B and individual efforts are not acknowledged in this setting of DM, there is a risk that she intentionally hinders the job thus causing a delay to enable her team win the reward.

\section{Method and Research Settings}

This research builds on our previous studies conducted in [15, 20,21], which include interviews and open-ended surveys with experts, managers, and end-users in the domain of DM. This resulted in identification of various situations where ad-hoc implementation of DM could lead to the creation of negative effect and issues amongst employees. Our analysis resulted in six representative scenarios in which an ad-hoc implementation of DM could create negative impact and issues amongst team members. In order to discover the resolution strategies that could help to resolve the 
negative effect in such scenarios, a four-stage study shown in Table 1 was designed for this purpose.

In the first stage, further analysis of the results from the previous studies was carried out. It was informed by the literature using the main theories in group dynamics [22], group cohesion and development [23], social identity theory [24], group conflict theory [25], change management [26], occupational psychology [27] and prosocial behaviour [28]. Various situations were also investigated where ad-hoc implementations of DM could create negative effect amongst the social actors within the workplace which resulted in six scenarios according to the main theories in conflict resolution. This helped us to generate around seventeen strategies which are intended to help to resolve negative effect in teamwork.

In the next stage of the study, and in order to refine these strategies, we conducted interviews with ten interviewees, including four experts in the domain of psychology; two in computing and social informatics and four from related workplaces of whom two were practitioners and two were managers where DM techniques have been implemented. This helped us to elaborate on our initial set and devise a final set of negative effect management strategies. All of the interviews were recorded and transcribed. The interviews followed a semi-structured style in order to refine with each participant the most appropriate strategies that could help reduce the likelihood of the negative effect, alleviate the adverse effect or resolve it for each scenario. This resulted in 22 strategies which could help in managing teamwork negative impact in relation to DM.

Table 1. Research method stages

\begin{tabular}{|c|c|c|c|}
\hline $1^{\text {st }}$ Stage & $2^{\text {nd }}$ Stage & $3^{\text {rd }}$ Stage & $4^{\text {th }}$ Stage \\
\hline Previous studies & Analysis & Interviews & Focus group \\
\hline $\begin{array}{l}\text { The work done in: } \\
\text { - DM obstacles and } \\
\text { ethical issues } \\
\text { identification [15] } \\
\text { - DM persona } \\
\text { aspect [21] } \\
\text {-DM modelling and } \\
\text { structuring aspect } \\
\text { [20] }\end{array}$ & $\begin{array}{l}\text { - The authors generated } \\
\text { six scenarios based on } \\
\text { stage } 1 . \\
\text { - The authors defined } \\
\text { resolution strategies } \\
\text { based on: } \\
\text { - Group dynamics } \\
\text { - Group cohesion } \\
\text { - Social identity } \\
\text { - Conflict theory } \\
\text { - Change management } \\
\text { - Occupational } \\
\text { psychology } \\
\text { - Prosocial behaviour } \\
\text { - Social norms }\end{array}$ & $\begin{array}{l}\text { The authors refine } \\
\text { the strategies } \\
\text { through interviews: } \\
\text { - Two experts in } \\
\text { computing and } \\
\text { social informatics } \\
\text { - Four experts in } \\
\text { psychology and } \\
\text { cyber-psychology } \\
\text { - Two practitioners } \\
\text { - Two managers }\end{array}$ & $\begin{array}{l}\text { The authors } \\
\text { refine the results } \\
\text { from } 1^{\text {st }}, 2^{\text {nd }} \text { and } \\
3^{\text {rd }} \text { stage via a } \\
\text { focus group } \\
\text { with } \\
\text { participants of a } \\
\text { multi- } \\
\text { disciplinary } \\
\text { background (see } \\
\text { Table 2) }\end{array}$ \\
\hline
\end{tabular}

In the final stage, the strategies were classified using a focus group with seven participants with relevant expertise. The participants were familiar with DM and came 
from diverse domains (see Table 2). Participants were familiarised with the context by means of presentation before the session, the six scenarios were provided as a hard copy, a facilitator explained the scenarios and answered questions during the session, and separate sheets of paper were provided to write down participants' ideas. The session was held in two parts in order to qualify the final results of these strategies. In the first part, the participants were given the scenarios and asked to brainstorm and suggest ideas, strategies and concepts which could help to manage the negative effect in each one. In the second part, they were given a list of possible resolution strategies and the description for each scenario, and then they were asked to provide their perception on these strategies and how they could help to resolve the negative effect on teamwork in relation to DM.

Table 2. $4^{\text {th }}$ stage focus group participants

\begin{tabular}{|l|l|}
\hline Participants & \multicolumn{1}{c|}{ Research Background } \\
\hline F & Facilitator (one of the authors) \\
\hline P1,P2 & $\begin{array}{l}\text { Requirements Engineering, Computers in Human behaviour and Cyber } \\
\text { Psychology }\end{array}$ \\
\hline P3,P4 & Human Factors and User Testing \\
\hline P5 & Usability and Human Computer Interaction \\
\hline P6 & Machine Intelligence and User Modelling \\
\hline P7 & Business Management \\
\hline
\end{tabular}

\section{$4 \quad$ Results}

In this section, we report and discuss the results of the study which revolve around two main aspects. In 4.1 we will describe the first aspect which concerns our proposed strategies to reduce DM side-effects on team work. In 4.2 we address the second aspect which is about categorising the strategy according to various development and management styles and phases.

\subsection{Strategies for Managing DM Negative Effects on Teamwork}

- Commitment: this strategy is based on the members' agreement and adoption of the choices and actions characterizing how DM is going to operate. This could be achieved by running a negotiation session to discuss views and exchange offers. This would then lead to maximized ownership of DM solution and accepting it.

- Common ground rules: this strategy is based on deriving and enforcing rules that articulate the set of acceptable behaviours in relation to DM, in order to facilitate the development of the use of DM within the organisation. Examples of such rules include showing respect for others, appropriate ways in which to express oneself, allowing everyone to 'have a say', openness to different views and confidentiality. This would help to manage and govern the work environment and also reduce the chance of negative effect in the workplace.

- Facilitator: this strategy plays an important role in facilitating the design sessions of DM, including running negotiation sessions, helping people to understand the 
common objectives of a group and assisting groups to set the common rules of conduct in an effective work environment supported by DM.

- Anonymity: the core idea of this strategy is to give opinions or ratings of colleagues or managers in an anonymous way. This could help make the work collaboration environment open. For example, this technique could help with the second case described in Section 2 to rate the employees' performances and prevent them from sabotaging the teamwork performances.

- Voting: this strategy helps to reach a decision in a facilitated session. When multiple choices are available amongst DM stakeholders in the design process, the facilitator could use a voting technique to try to meet the concerns of team members in a democratic and more acceptable style.

- Norms: this technique is based on having a clear understanding of what the organisational culture is, e.g. normal social behaviours. This could help to reduce the likelihood of negative effects within rewarding system environments. For example, an organisation may have a norm of senior managers publicly acknowledging successes of team members in monthly team meetings. A new DM based reward system such as a leader board may aim to serve the same basic function of highlighting success within the team, but the departure from the previously established norm of face to face social approval may cause resentment in team members.

- Transparency: this strategy means allowing everyone to see other's performances in DM system. Although some participants involved in this study agreed on the importance of this strategy to resolve DM negative effects, others mentioned that "it should be designed carefully to avoid clustering high performances workers and those of the lower performances".

- Rotations sensitivity: this strategy is based on allocating people randomly within DM system so that cliques and rivalries are not created. This could help to eliminate a negative effect caused by workers only supporting their close colleagues to win any reward.

- Get everyone involved: this strategy encourage people in different roles to become involved in a discussion to decide behaviours and penalties for their DM system.

- Story telling: the core idea of this strategy is to identify negative effect by asking people to present a situation in a story. A manager involved in our study noticed that "when we have a conflict in our company I sometimes go out for walk with some of my staff and ask them to tell the situation in a story, this can help to determine the source of the conflict".

- Round robin: this strategy aims to pass the discussion between workers one by one to ensure everyone gives their ideas individually. This would help to ensure the equality amongst workers involved in DM system and ensure everyone gets a chance to speak.

- External party: this strategy proposes to use an external authority or expert to check workers' performances and to resolve negative effects which might arise in relation to DM.

- Non-contentious bargaining: this strategy encourages team members to control their emotions in a professional way, such as "counting to ten" before taking an 
action, writing down their concerns carefully in an email or letter with a calm manner [29]. This strategy can be used to reduce negative effects of DM without causing additional side-effects. For example, a group leader may only acknowledge top performing members of a group, via badges and status, despite the remaining group members performing their roles adequately. By expressing their concerns in calm, reasoned (i.e. non-contentious) manner the group members may be able to reach agreement with the group leader on how a DM system can be changed to the mutual benefit of all involved [30].

- Reward for helping others: this strategy is related to prosocial theory, in which users can be rewarded for supporting others. This could be used in any DM to encourage collaborative teamwork such as, encouraging workers who always appear in the top list of a leader-board by rewarding them when helping their lower performances colleagues to appear in the leader-board.

- Acknowledgement of individual efforts: in some DM situations negative effect on teamwork might arise when individual efforts are not equal. This could arise when some workers rely on others to finish a task and is based on the concept of social loafing, so this strategy could help to inspire individuals to engage in group tasks to completion.

- Observation strategies: various strategies using different techniques to help to observe DM teamwork environments include:

- Auditing: means checking individual performances, e.g. giving a quantifiable task and assuming people will also respect quality. Although the auditing technique can help to resolve negative effect on teamwork, one practitioner said "it should be used in a very careful style to prevent introducing another conflict".

- Random monitoring: the idea of this technique is to keep workers ready all of the time as their performances might be monitored at any time.

- Peer-rating: this technique means that colleagues can rate each other's efforts and might be checked at any time to avoid a biased evaluation.

- Member checking: this strategy utilises a sample member in order to analyse the eventual DM result after finishing the task.

- Managerial level monitoring: in this strategy managers take the responsibility to check workers' performances in DM workplace.

- Self-assessment: users assess their own performances, and this might be checked by managers at any time.

- Regular meetings: involving teamwork members in regular meetings, e.g. weekly, monthly or annually would help managers to remain updated with the current use of DM system.

\subsection{The Categorization of Strategies for DM Management in Teamwork}

From the analysis of the interviews and the focus group, it was possible to extract the need for different ways to represent these strategies to resolve negative effect on teamwork within DM workplaces. As a result, three main categorises for better representation of these strategies. These are resolution strategies development aspect, resolution strategies enactment aspect and resolution strategies usage aspect. The 
concept map for each aspect is represented in Figures 1, 2 and 3 to illustrates the main characteristics of these aspects and provide examples of strategies which could help to manage the negative impact in teamwork related to DM.

\section{Resolution Strategies: Development Stage Aspect}

The analysis of our empirical studies shows that there are some strategies applicable at the design time of DM, whilst others might be used in real-time when DM is being used in the workplace and finally some may be useful for both.

- At the design stage: it seems that strategies would help in setting up agreements, rules and structuring the general guidelines of DM system can be fitted at this stage. For example, some practitioners and psychologists mentioned that we should get everyone involved in a discussion making session at design stage and make them committed to the design of their DM. However, others suggested that having a sample of employees could be a help since in large organisations, where the number of employees is very high and it is impossible to engage everyone in the design stage. Moreover, the majority of interviewees agreed on having common ground rules at the early stages and asking users to agree on DM rules, which could help reduce the negative effect which might occur during the actual work.

- At the run-time: the analysis results suggest that strategies with characteristics like observing and controlling the environments would fit more into this stage. For instance, strategies such as auditing, random monitoring, peer-rating and member-checking could help in teamwork to observe the quality of the work and to control and resolve negative effects when they happen.

Fig. 1. Concept map of resolution strategies from development stage perspective

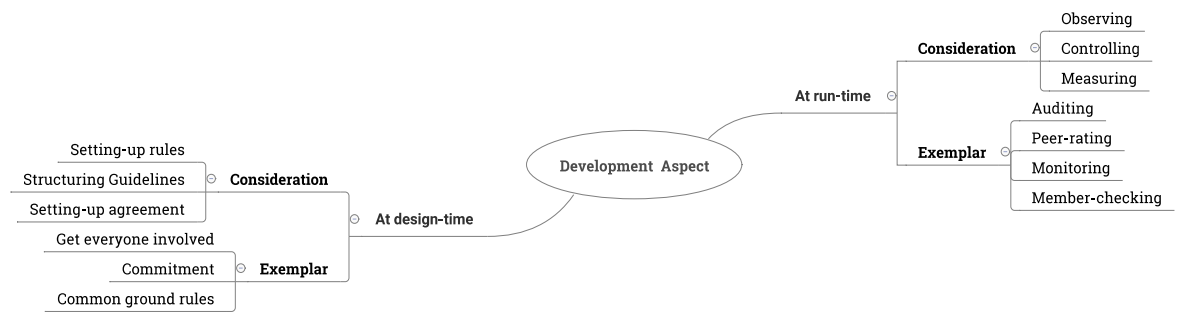

\section{Resolution Strategies: Enactment Aspects}

In order to apply these strategies within a teamwork places there could be different approaches giving more effective implementation results. These have been identified as collaborative, moderated and directive, as explained below. However, there is a difficulty in the suitability of these strategies as some of them might apply for more than one approach and others cannot be easily decided. For example, a strategy like Peer-rating can fit as collaborative amongst workers; however it might have a valuable result when it's moderated by managers or project leaders. 
- Collaborative approach: our studies suggest that strategies which have mutual benefits e.g. reward for helping others is better to be decided collaboratively. Moreover, strategies which are subjective and require decision making would fit in such an approach. For instant, round robin strategy where workers can engage in a discussion equally to decide to what extent they should cooperate with each other in the task and how DM could be used to measure their performances would be preferable to be implemented in a collaborative way.

- Moderated approach: this approach would help with strategies which are complex and where workers are not able to steer the process to reach the consensus. External authorities or experts work collectively with managers to set up the strategy and moderate the interaction. For example, in the external party strategy managers work together with external consultants to decide the effective way to manage the strategy to resolve the negative effect within DM.

- Directive approach: the nature of some strategies which are based on explicit polices, with well-defined directions and clear objective measures can be operated effectively. For example, some participants suggested that managers can play the key roles in resolving negative effect within some scenarios through leading the observing or auditing process.

Fig. 2. Concept map for resolution strategies from enactment perspective

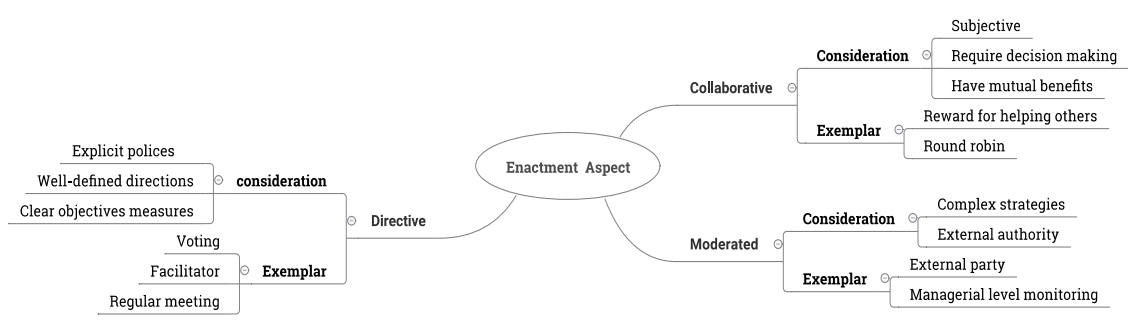

\section{Resolution Strategies: Usage Aspect}

The usage aspect is related to the different ways these strategies can be used to manage negative effect on teamwork. As a general principle, the participants used this labelling to categorise characteristics of the effect of these strategies. They assume that some of them would help to alleviate the negative effect when it is impossible to resolve it. Moreover, these strategies are not mutually exclusive strategies they might be used for more than one aspect e.g. detection and resolution at the same time whether at design stage of DM or in real-time at a workplace.

- Detection strategies: it seems from the characteristics of some strategies that they would help more to identify where the limitations or weaknesses are in DM more than resolving negative effect. For example, the observation strategies have the checking and inspection features which could help more to identify where the negative effect originate. 
- Resolution strategies: the main mission of these strategies is to help to resolve negative effect on teamwork. Strategies which allow making attractive offers, the exchange of interests and rewarding agreement would fit more as resolution strategies e.g. rewards for helping others and rewards for individual contributions. Applying such strategies to the second case in section 2 above would help to prevent Alice from sabotaging the teamwork in team $A$ and will encourage her to involve in the teamwork.

- Alleviation strategies: in some cases, the negative effect cannot be resolved. Thus these types of strategies which support self-recognition and unexpected intervention actions could help to reduce the negative effect. For example, some experts commented that strategies such as random monitoring or anonymity cannot help to resolve conflict, but it might assist to reduce the negative effect.

- Prevention strategies: strategies based on specifying objectives, timeframes and policy tools would play important roles in reducing the likelihood of negative effect from happening in DM work environment. For example, strategies used at the early stages of DM, such as having common ground rules and asking users for commitments could help to reduce negative effect arising in workplace.

Fig. 3. Concept map for resolution strategies from usage perspective

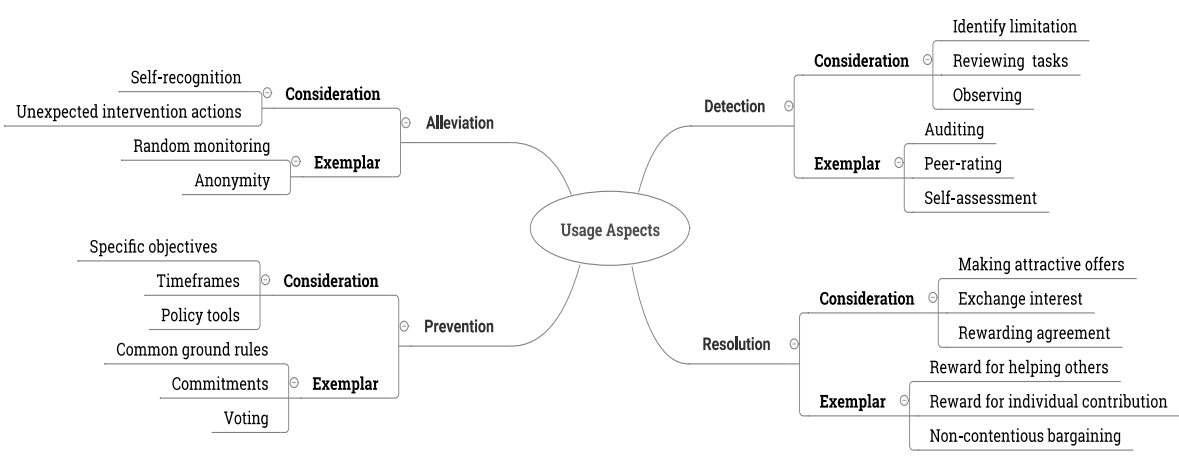

As an example in teamwork negative effect scenario one in section 2, we may apply strategies like common ground rules and commitment at the design of DM stage and ask the front-end development team to commit on rules such as, everyone should respect all the team members and act with them in similar manner. At the run-time stage we could apply one of the observation strategies e.g. auditing or managerial level monitoring to check whether workers respect that commitment. In addition, if we detect negative effect happening in this group we might use a strategy like reward for helping others to encourage high performance workers to support lower performance which would help to resolve the negative impact in this scenario.

\section{Conclusion and Future Work}

In spite of the increasing use and success of DM tools to persuade users to be more motivated and engaged, further studies should focus on how to resolve negative issues 
and side-effects related to its use in the workplace. Amongst various problems which could happen in workplaces, such as a lowering of quality or creation of tension, this paper focused on strategies to manage negative effect on teamwork as one of the significant risks of introducing DM elements into the work environment. We explored the resolution strategies from both psychological and management perspectives, which could help to introduce DM into the work environment in a healthier and coherent way. Our study led to 22 teamwork negative effect management strategies which could help to minimize workplaces negative impact related to DM. We also categorized these strategies into three main aspects based on their goal, stage and purpose of use.

In future work we will further investigate each of the three stages from various stakeholders' perspectives. In particular, we plan to study the use of participatory design [31] in order to engage team members in the development of DM itself as it can incorporate a wide range of the strategies and be by itself a powerful mechanisms for an agreeable and effective DM. We also plan to use negotiation theory as part of the construction of DM solutions so that the rewards can be agreed for tasks involving different stakeholders. A further validation of the suitability and constraints on the proposed strategies will be achieved via practical case study.

\section{Acknowledgment}

The research was supported by Bournemouth University through the PGR Development Fund and the FP7 Marie Curie CIG grant (the SOCIAD project). We also thank the participants in our studies for their valuable contributions.

\section{References}

1. Fogg, B.J.: Persuasive Technology: Using Computers to Change What We Think and Do (Interactive Technologies). (2002).

2. Deterding, S., Dixon, D., Khaled, R., Nacke, L.: From game design elements to gamefulness - defining "gamification". Presented at the The th International Academic MindTrek Conference Envisioning Future Media Environments, Tampere September (2011).

3. Ahn, von, L.: Games with a Purpose. IEEE Computer. 39, 92-94 (2006).

4. Magerkurth, C., Cheok, A.D., Mandryk, R.L., Nilsen, T.: Pervasive games - bringing computer entertainment back to the real world. Computers in Entertainment. 3, 4-4 (2005).

5. King, D., Greaves, F., Exeter, C., Darzi, A.: "Gamification": Influencing health behaviours with games. Journal of the Royal Society of Medicine. 106, 76-78 (2013).

6. Spanakis, E.G., Santana, S., Ben-David, B.: Persuasive technology for healthy aging and wellbeing. Wireless Mobile Communication and Healthcare (Mobihealth), 2014 EAI 4th International Conference on (pp. 23-23). IEEE. (2014).

7. Lacroix, J., Saini, P., Goris, A.: Understanding user cognitions to guide the tailoring of persuasive technology-based physical activity interventions. 4th international conference on persuasive technology. 350, (2009).

8. Herzig, P., Ameling, M., Schill, A.: A Generic Platform for Enterprise Gamification. Presented at the 2012 Joint Working IEEE/IFIP Conference on Software Architecture (WICSA) \& European Conference on Software Architecture (ECSA).

9. Beenen, G., Ling, K.S., Wang, X., Chang, K., Frankowski, D., Resnick, P., Kraut, R.E.: Using social psychology to motivate contributions to online communities. CSCW. 212-221 
(2004).

10. Simões, J., Redondo, R.D., Vilas, A.F.: A social gamification framework for a K-6 learning platform. Computers in Human Behavior. 29, 345-353 (2013).

11. Fogg, B.J.: Creating persuasive technologies - an eight-step design process. PERSUASIVE. 350, 1 (2009)

12. Nicholson, S.: A user-centered theoretical framework for meaningful gamification. Games+ Learning+ Society. (2012).

13. Consolvo, S., Klasnja, P.V., McDonald, D.W., Landay, J.A.: Goal-setting considerations for persuasive technologies that encourage physical activity. PERSUASIVE. 350, 1 (2009).

14. Gram-Hansen, S.B.: The EDIE Method - Towards an Approach to Collaboration-Based Persuasive Design. In: Persuasive Technology. pp. 53-64. Springer International Publishing, Cham (2016).

15. Shahri, A., Hosseini, M., Phalp, K., Taylor, J., Ali, R.: Towards a Code of Ethics for Gamification at Enterprise. PoEM. 197, 235-245 (2014).

16. Nicholson, S.: A user-centered theoretical framework for meaningful gamification. Games+ Learning+ Society. (2012).

17. Timmer, J., Kool, L., van Est, R.: Ethical Challenges in Emerging Applications of Persuasive Technology. PERSUASIVE. (2015).

18. Chidambaram, L., Tung, L.L.: Is Out of Sight, Out of Mind? An Empirical Study of Social Loafing in Technology-Supported Groups. Information Systems Research. 16, 149-168 (2005).

19. Bajdor, P., Dragolea, L.: The gamification as a tool to improve risk management in the enterprise. " Annales Universitatis Apulensis: Series Oeconomica 13.2. (2011).

20. Shahri, A., Hosseini, M., Phalp, K., Taylor, J., Ali, R.: Exploring and Conceptualising Software-Based Motivation Within Enterprise. PoEM. 267, 241-256 (2016).

21. Shahri, A., Hosseini, M., Almaliki, M., Phalp, K., Taylor, J., Ali, R.: Engineering softwarebased motivation - A persona-based approach. RCIS. 2016-August, 1-12 (2016).

22. Forsyth, D.R.: An introduction to group dynamics. Thomson Brooks/Cole (1983).

23. Tuckman, B.W., Jensen, M.A.C.: Stages of Small-Group Development Revisited. Group \& Organization Management. 2, 419-427 (1977).

24. Ellemers, N., De Gilder, D., Haslam, S.A.: Motivating individuals and groups at work: a social identity perspective on leadership and group performance. Academy of Management Review. 29, 459-478 (2004).

25. Forsyth, D.: Group Dynamics. Cengage Learning (2009).

26. Hayes, J.: The Theory and Practice of Change Management. Palgrave Macmillan (2014).

27. Ashforth, B.E., Mael, F.: Social Identity Theory and the Organization. Academy of Management Review. 14, 20-39 (1989).

28. Denham, S.A.: Social Cognition, Prosocial Behavior, and Emotion in Preschoolers: Contextual Validation. Child Development. 57, 194-2 (1986).

29. McGillicuddy, N.B., Pruitt, D.G., Syna, H.: Perceptions of Firmness and Strength in Negotiation. Personality and Social Psychology Bulletin. 10, 402-409 (1984).

30. Forgas, J.P.: On feeling good and getting your way: Mood effects on negotiator cognition and bargaining strategies. Journal of Personality and Social Psychology. 74, 565-577 (1998).

31. Kensing, F., Blomberg, J.: Participatory Design: Issues and Concerns. Computer Supported Cooperative Work (CSCW). 7, 167-185 (1998). 